\section{Maristela Cavalheiro}

Tamborindeguy França'

Elsa Regina Justo Giugliani"

Luciana Dias de Oliveira"'

Enilda Maria Lara Weigert ${ }^{\mathrm{IV}}$

Lilian Cordova do Espirito

Santo $^{\mathrm{v}}$

Celina Valderez Köhlervı

Ana Lucia de Lourenzi Bonilhav

Hospital Criança Conceição. Porto Alegre RS, Brasil

Departamento de Pediatria e Puericultura. Faculdade de Medicina. Universidade Federal do Rio Grande do Sul (UFRGS).

Porto Alegre, RS, Brasil

III Escola de Nutrição. Pontifícia Universidade Católica do Rio Grande do Sul. Porto Alegre, RS, Brasil

Iv Hospital Moinhos de Vento. Porto Alegre, RS, Brasil

Departamento de Enfermagem MaternoInfantil. Escola de Enfermagem. UFRGS Porto Alegre, RS, Brasil

" Coordenadoria Regional de Saúde. Secretaria de Estado de Saúde do Rio Grande do Sul. Porto Alegre, RS, Brasil

Correspondência | Correspondence: Maristela C T França

Av. Itajaí, 191/201

90470-140 Porto Alegre, RS, Brasil

E-mail: maristela@franca.bio.br

\section{Uso de mamadeira no primeiro mês de vida: determinantes e influência na técnica de amamentação}

\section{Bottle feeding during the first month of life: determinants and effect on breastfeeding technique}

\section{RESUMO}

OBJETIVO: Analisar a incidência e os determinantes do uso de mamadeira no primeiro mês de vida e possíveis efeitos dessa prática na técnica de amamentação.

MÉTODOS: Estudo transversal aninhado em uma coorte contemporânea de Porto Alegre, RS, de junho a novembro de 2003. Durante um mês foram acompanhados 211 pares de mãe e criança. A influência do uso de mamadeira sobre a técnica de amamentação foi avaliada comparando-se as freqüências de cinco itens desfavoráveis ao posicionamento mãe/criança e três itens desfavoráveis à pega da criança; e as médias do número de itens desfavoráveis entre as duplas que iniciaram o uso mamadeira no primeiro mês e as que não o fizeram. A regressão logística estimou o grau de associação das variáveis com os desfechos, utilizando modelo hierarquizado.

RESULTADOS: Aos sete dias, $21,3 \%$ das crianças usavam mamadeira e, aos 30 dias, 46,9\%. Coabitação com a avó materna esteve associada com uso de mamadeira tanto aos sete quanto aos 30 dias. Também estiveram associados ao uso de mamadeira aos sete dias: a mãe ser adolescente e trauma mamilar na maternidade. Os outros dois fatores associados ao uso de mamadeira aos 30 dias foram trauma mamilar aos sete dias e uso de chupeta aos sete dias. Não houve associação entre técnica de amamentação ensinada na maternidade e uso de mamadeira, mas aos 30 dias, crianças que usavam mamadeira apresentaram técnica menos adequada às que sugavam só no peito.

CONCLUSÕES: Os resultados mostram que a mamadeira foi bastante utilizada no primeiro mês de vida, principalmente: em crianças com mães adolescentes e com trauma mamilar, cujas avós maternas estavam presentes no domicílio e que faziam uso de chupeta. Além dos efeitos negativos já conhecidos, a mamadeira pode influenciar negativamente a técnica de amamentação.

DESCRITORES: Alimentação Artificial. Aleitamento Materno. Cuidado do Lactente. Fatores Epidemiológicos. Estudos Transversais. Mamadeira. 


\section{ABSTRACT}

OBJECTIVE: To assess incidence rate and determinants of bottle feeding during the first month of life, and its potential effects on breastfeeding technique.

METHODS: A nested cross-sectional study was conducted in a contemporary cohort in the city of Porto Alegre, Southern Brazil, between June and November 2003. A total of 211 pairs of healthy mothers and infants were followed up for a month. The effect of bottle feeding on breastfeeding technique was assessed by comparing five items unfavorable to mother-infant positioning and three items unfavorable to infant latch-on; and the average number of unfavorable items between the pairs who started bottle feeding in the first month of life and those who did not. A logistic regression analysis was carried out according to a hierarchical model.

RESULTS: By Day 7, 21.3\% of infants were bottle-fed and $46.9 \%$ were bottle-fed by Day 30 . Living with maternal grandmother was associated with bottle feeding at Days 7 and 30. Maternal age $<20$ years, and nipple trauma at the maternity hospital were also associated with bottle feeding at Day 7 . Other factors associated with bottle feeding at Day 3 were pacifier use at Day 7 and nipple trauma at Day 7. There was no association between the breastfeeding technique taught at the maternity hospital and subsequent bottle feeding. However, at Day 30, the breastfeeding technique was found to be more adequate among exclusively breast-fed infants than those who were also bottle fed.

CONCLUSIONS: The study results showed that bottle feeding was quite widespread in the first month of life, mainly in infants born to teenage mothers, with nipple trauma, whose maternal grandmothers were living in the same household, and who were using pacifiers. Besides the already recognized negative effects, bottle feeding may negatively affect breastfeeding technique.

DESCRIPTORS: Bottle Feeding. Breast Feeding. Infant Care. Epidemiologic Factors. Cross-Sectional Studies. Bottle feeding.

\section{INTRODUÇÃO}

$\mathrm{O}$ aleitamento materno favorece o crescimento e o desenvolvimento da criança, tanto por suas características nutricionais, imunológicas e psicológicas, quanto por possibilitar o crescimento harmonioso da face, promovendo a maturação das funções do sistema estomatognático. ${ }^{11}$ A amamentação é recomendada de forma exclusiva até o sexto mês de vida e de forma complementada até dois anos ou mais, ${ }^{a}$ tornando desnecessário o uso de mamadeiras com qualquer tipo de líquido. No entanto, pesquisa de âmbito nacional ${ }^{b}$ revelou que a utilização da mamadeira é uma prática bastante freqüente em $62,8 \%$ das crianças menores de um ano no Brasil.

Desde meados do século passado, vários estudos mostram que o uso da mamadeira interfere nas funções de mastigação, sucção e deglutição, sendo capaz de alterar a musculatura dos órgãos fonoarticulatórios e a oclusão dentária. ${ }^{6,22}$ Além disso, a mamadeira é considerada importante fonte de contaminação das crianças e um dos fatores de risco para a ocorrência de otite média. ${ }^{16}$ Quando introduzida precocemente, acredita-se que a mamadeira possa gerar "confusão de bicos", devido às diferenças existentes entre a sucção na mama e no bico artificial. ${ }^{10}$

Ante a escassez de pesquisas sobre determinantes do uso de mamadeira, faz-se necessário recorrer a estudos sobre os determinantes do aleitamento materno exclusivo, pois outros alimentos são geralmente oferecidos

\footnotetext{
a Espirito Santo LC. Fatores associados à interrupção precoce do aleitamento materno exclusivo e influência do padrão de aleitamento materno no primeiro mês de vida na duração da amamentação [tese de doutorado]. Porto Alegre: Faculdade de Medicina da UFRGS; 2006 [citado 2007 ago 20]. Disponível em: http://www.dominiopublico.gov.br/download/texto/cp024634.pdf

b Brasil. Ministério da Saúde, Secretaria de Políticas de Saúde, Área de Saúde da Criança. Prevalência de aleitamento materno nas capitais brasileiras e no Distrito Federal. Brasília; 2001.
} 
em mamadeira. Vários estudos brasileiros identificaram determinantes da interrupção precoce do aleitamento materno exclusivo (AME), tais como: uso de chupeta, baixa escolaridade e idade maternas, primiparidade, cesárea $^{\mathrm{a}} \mathrm{e}$ conselho das avós da criança para a utilização de suplementos, ${ }^{15}$ entre outros. Estudos internacionais têm descrito associação entre técnica inadequada de amamentação e menor duração do aleitamento materno exclusivo. ${ }^{3,12}$ Por outro lado, crianças com alimentação mista (peito e mamadeira) podem desenvolver uma técnica incorreta de sucção no seio. ${ }^{13}$ Esse tópico tem sido pouco explorado na literatura, não se conhecendo, por exemplo, a influência do uso de mamadeira no posicionamento mãe/criança e na pega da criança durante a amamentação, itens considerados importantes para o sucesso do aleitamento materno.

O presente trabalho teve por objetivo analisar fatores associados à freqüência do uso de mamadeira no primeiro mês de vida e possíveis efeitos dessa prática na técnica de amamentação.

\section{MÉTODOS}

Foi realizado estudo transversal aninhado em coorte contemporânea com mães e recém-nascidos residentes em Porto Alegre (RS), atendidos em maternidade, no período de junho a novembro de 2003. Essa maternidade é credenciada como Hospital Amigo da Criança, contando com três consultoras em lactação certificadas pelo International Board of Lactation Consultant Examiners, para acompanhamento de duplas mãe-criança. Foram incluídas no estudo duplas que estavam em alojamento conjunto, cujos recém-nascidos tinham peso de nascimento igual ou acima de $2.500 \mathrm{~g}$, eram não gemelares e tinham iniciado a amamentação. Foram excluídos da amostra os pares que necessitaram se separar ao longo do primeiro mês de vida e que tiveram algum problema que contra-indicasse a amamentação.

Para o cálculo amostral foram utilizados os seguintes parâmetros: $:^{12} \alpha=5 \%$ e $\beta=20 \%$; prevalência de técnica de amamentação insatisfatória em crianças não expostas à mamadeira: $35 \%$; diferença mínima das prevalências do desfecho entre expostos e não-expostos: $20 \% .^{9,19}$ Assim, estimou-se amostra mínima de 206 duplas mãe-criança.

Das 233 duplas selecionadas para o estudo, $12(5,1 \%)$ não participaram por recusa da mãe, $9(3,9 \%)$ foram perdidas ao longo do acompanhamento por não terem sido localizadas e $1(0,4 \%)$ foi perdida por registro incompleto dos dados, resultando em amostra final de 211 duplas.
A amostra foi selecionada entre junho e novembro de 2003, com sorteio diário de duas duplas, incluindo os finais de semana. Após assinatura do termo de consentimento informado, iniciava-se a coleta de dados por meio de entrevistas semi-estruturadas, realizada em três fases, e observação da mamada. A primeira, na maternidade, entre o segundo e o terceiro dia após o nascimento da criança, as mães foram entrevistadas visando-se obter informações relativas às características sociodemográficas e aspectos relacionados ao acompanhamento pré-natal, parto e experiência prévia com amamentação. Na segunda, após a entrevista, foi avaliada a técnica de amamentação por meio de observação de uma mamada completa, em que foram pesquisados cinco itens desfavoráveis ao posicionamento mãe/criança (mãe com ombros tensos, não relaxados; criança distante da mãe; cabeça e tronco da criança não alinhados; queixo da criança não toca o seio; e criança não apoiada firmemente, somente ombros e cabeça apoiados) e três itens desfavoráveis à pega (boca pouco aberta; lábio inferior não evertido; e pega não assimétrica, com mais aréola visível na parte inferior da boca do que na superior). ${ }^{17}$ A avaliação na maternidade foi realizada por três pesquisadoras treinadas e padronizadas, com índice de concordância de $90 \%$. Na terceira fase, as mamas foram examinadas para a detecção de trauma mamilar (fissuras, escoriações, equimoses, marcas/bolhas).

Nas segunda e terceira fases da coleta, aos sete e 30 dias de vida da criança, no domicílio da mãe, foram coletadas as seguintes informações: alimentação da criança, uso de mamadeira e de chupeta e dor/trauma mamilar. Aos sete dias, as informações foram colhidas por estudantes de medicina e enfermagem, previamente treinados quanto à aplicação da entrevista e avaliação das mamas. A observação da mamada aos 30 dias foi realizada pela mesma pesquisadora que fez a observação na maternidade, aplicando os mesmos critérios utilizados na primeira fase da coleta de dados. Considerou-se em aleitamento materno exclusivo a criança que recebia somente leite materno sem ingerir outro líquido ou sólido, exceto vitaminas, complementos minerais ou medicamentos. ${ }^{5}$

Utilizou-se o programa EpiInfo versão 6.0 para processamento dos dados, com dupla digitação. Para as análises estatísticas foi utilizado o programa SPSS versão 10.0 .

Para medir a força das associações entre as variáveis de interesse foi realizada regressão logística multivariada, com a utilização de modelo hierarquizado em quatro etapas.

Na primeira, foram introduzidas as variáveis escolaridade, idade e cor da pele da mãe e sexo da criança. $\mathrm{Na}$ segunda etapa, foram incluídas coabitação com avó

\footnotetext{
a Espirito Santo LC. Fatores associados à interrupção precoce do aleitamento materno exclusivo e influência do padrão de aleitamento materno no primeiro mês de vida na duração da amamentação [tese de doutorado]. Porto Alegre: Faculdade de Medicina da UFRGS; 2006 [citado 2007 ago 20]. Disponível em: http://www.dominiopublico.gov.br/download/texto/cp024634.pdf
} 
materna, coabitação com avó paterna, coabitação com companheiro, paridade e número de consultas prénatais. Duração da amamentação de filhos anteriores e tipo de parto foram incluídos na terceira e na quarta etapas foram acrescentados uso de chupeta aos sete dias, ingurgitamento mamário aos sete dias e trauma mamilar na maternidade e aos sete dias. Para o controle de possíveis fatores de confusão, foram mantidas as variáveis que atingiram nível de significância $\mathrm{p} \leq 0,20$ quando introduzidas no modelo. As variáveis que apresentaram nível de significância $\mathrm{p}<0,05$ permaneceram no modelo final. A medida de associação utilizada para indicar quantas vezes a prevalência do desfecho foi aumentada por influência dos fatores foi a odds ratio.

Para quantificar a qualidade do posicionamento mãe/ criança e da pega da criança, calculou-se o número de itens desfavoráveis encontrados na avaliação da mamada. Assim, quanto menor o número de itens desfavoráveis, melhor a técnica. Foi utilizado o teste $\mathrm{t}$ de Student nas comparações das médias de itens desfavoráveis entre os grupos e o teste $t$ de Student para amostras pareadas, nas comparações intragrupos. Adotou-se $\mathrm{p}<0,05$ como nível crítico e intervalos de confiança de $95 \%$.

O estudo foi aprovado pela Comissão de Pesquisa e Ética em Saúde do Grupo de Pesquisa e Pós-Graduação do Hospital de Clínicas de Porto Alegre.

\section{RESULTADOS}

A Tabela 1 apresenta as características dos participantes do estudo. A quarta parte das mães era adolescente e a maioria era de cor branca, com oito anos ou mais de escolaridade. Todas as mães realizaram acompanhamento pré-natal e a maioria teve seis consultas ou mais. Quase metade das mães era primípara e a maioria coabitava com o companheiro. Praticamente um terço da amostra coabitava com avós maternas da criança e um quinto, com avós paternas. Pouco menos de metade das mães apresentou trauma mamilar na maternidade ou aos sete dias pós-parto, e um terço delas apresentou ingurgitamento mamário aos sete dias. No final da primeira semana de vida, um terço das crianças já utilizava chupeta.

Dos 211 pares acompanhados, oito interromperam a amamentação antes de completar um mês pós-parto. Assim, foi possível a observação da mamada aos 30 dias em 203 pares. Aos sete dias, 21,3\% das crianças usavam mamadeira e, aos 30 dias, 46,9\%. O conteúdo oferecido por mamadeira aos sete e 30 dias para as crianças foi, respectivamente, chá $31(14,7 \%)$ e 78 $(37,0 \%)$; água $2(0,9 \%)$ e $20(9,4 \%)$; leite industrializado $8(3,8 \%)$ e $43(20,4 \%)$ e leite materno ordenhado $10(4,7 \%)$ e $8(3,8 \%)$.

A Tabela 2 apresenta o resultado da análise multivariada utilizada para testar associações entre os fatores em estudo e os desfechos. Das quatro variáveis introduzidas
Tabela 1. Características das duplas mãe-criança que participaram do estudo. Porto Alegre, RS, 2003. ( $\mathrm{N}=211)$

\begin{tabular}{lrl}
\hline Característica & N & $\%$ \\
\hline Idade da mãe $\geq 20$ anos & 160 & 75,8 \\
Mãe de cor branca & 148 & 70,1 \\
Escolaridade da mãe $\geq 8$ anos & 135 & 64 \\
Coabitação com companheiro & 175 & 82,9 \\
Coabitação com avó materna & 65 & 30,8 \\
Coabitação com avó paterna & 40 & 18,9 \\
Primeiro filho & 99 & 46,9 \\
Pré-natal $\geq 6$ consultas & 166 & 78,7 \\
Criança do sexo masculino & 110 & 52,1 \\
Parto vaginal & 151 & 71,6 \\
Duração média da amamentação & 78 & 37,1 \\
dos filhos anteriores $\geq 6$ meses* & & 43,6 \\
Trauma mamilar na maternidade & 92 & 46,9 \\
Trauma mamilar aos 7 dias & 99 & 47,4 \\
Uso de chupeta aos 7 dias & 100 & 34,6 \\
\hline Ingurgitamento mamário aos 7 dias & 73 &
\end{tabular}

* excluídas as primíparas

na primeira etapa da regressão, a pouca idade materna apresentou associação significativa com o uso de mamadeira aos sete dias. Nenhuma variável dessa fase apresentou associação significativa com o uso de mamadeira aos 30 dias. Dentre os cinco fatores em estudo introduzidos na segunda etapa, apenas a coabitação com a avó materna da criança apresentou associação significativa com os dois desfechos. Na terceira, nenhum fator introduzido se mostrou associado aos desfechos. $\mathrm{Na}$ quarta, das quatro novas variáveis introduzidas no modelo, a presença de trauma mamilar na maternidade mostrou-se associada ao uso de mamadeira aos sete dias, enquanto que uso de chupeta aos sete dias e presença de trauma mamilar aos sete dias foram os fatores associados ao uso de mamadeira aos 30 dias.

As médias do número de itens desfavoráveis ao posicionamento mãe/criança e à pega da criança na maternidade foram semelhantes entre o grupo de crianças que estava recebendo mamadeira aos 30 dias e o que só sugava no peito. No entanto, aos 30 dias, o grupo de crianças que recebeu mamadeira no primeiro mês apresentou maior número de itens desfavoráveis tanto no posicionamento quanto na qualidade da pega, indicando técnica menos adequada (Tabela 3). Comparando-se a média de número de itens desfavoráveis obtidos na maternidade com a obtida aos 30 dias, observa-se que as duplas cujas crianças não recebiam mamadeira mantiveram a média relacionada ao posicionamento $(p=0,469)$, mas esta diminuiu para a pega da criança $(\mathrm{p}<0,001)$. Já para o grupo que recebia mamadeira, não houve mudança significativa nas médias dos itens desfavoráveis no posicionamento $(p=0,073)$ e na pega $(p=0,129)$. 
Tabela 2. Associação entre os fatores em estudo e os desfechos uso de mamadeira aos 7 dias e uso de mamadeira aos 30 dias. Porto Alegre, RS, 2003. ( $\mathrm{N}=211)$

\begin{tabular}{|c|c|c|c|c|c|c|c|}
\hline \multirow{2}{*}{ Variável } & \multirow[b]{2}{*}{$\mathrm{N}$} & \multicolumn{3}{|c|}{$\begin{array}{l}\text { Uso de mamadeira aos } 7 \text { dias } \\
\qquad(\mathrm{N}=45)\end{array}$} & \multicolumn{3}{|c|}{$\begin{array}{l}\text { Uso de mamadeira aos } 30 \text { dias } \\
\qquad(\mathrm{N}=98)\end{array}$} \\
\hline & & $\begin{array}{c}\text { OR } \\
\text { ajustada* }\end{array}$ & IC 95\% & $\mathrm{p}$ & $\begin{array}{c}\text { OR } \\
\text { ajustada* }\end{array}$ & IC 95\% & $\mathrm{p}$ \\
\hline \multicolumn{8}{|l|}{ Primeira etapa } \\
\hline Escolaridade $<8$ anos & 76 & 0,83 & 0,$40 ; 1,70$ & 0,608 & 1,01 & 0,$57 ; 1,79$ & 0,981 \\
\hline Mãe adolescente & 51 & 2,44 & 1,$18 ; 5,04$ & $\mathbf{0 , 0 1 6 * *}$ & 1,88 & 0,$98 ; 3,62$ & $0,060^{* *}$ \\
\hline Cor da mãe branca & 148 & 0,57 & 0,$28 ; 1,15$ & $0,117^{* *}$ & 1,23 & 0,$66 ; 2,27$ & 0,514 \\
\hline Criança masculino & 110 & 1,05 & 0,$53 ; 2,08$ & 0,897 & 1,32 & 0,$76 ; 2,30$ & 0,325 \\
\hline \multicolumn{8}{|l|}{ Segunda etapa } \\
\hline Coabitação com avó materna & 65 & 2,58 & 1,$14 ; 5,87$ & 0,023* & 2,33 & 1,$18 ; 4,60$ & $0,015^{* *}$ \\
\hline Coabitação com avó paterna & 40 & 1,58 & 0,$58 ; 4,34$ & 0,374 & 0,77 & 0,$35 ; 1,68$ & 0,505 \\
\hline Coabitação com companheiro & 175 & 0,56 & 0,$22 ; 1,44$ & 0,228 & 1,09 & 0,$47 ; 2,53$ & 0,836 \\
\hline$<6$ consultas pré-natais & 42 & 0,75 & 0,$29 ; 1,88$ & 0,538 & 1,55 & 0,$75 ; 3,22$ & 0,237 \\
\hline Primiparidade & 99 & 1,19 & 0,$52 ; 2,72$ & 0,681 & 1,32 & 0,$69 ; 2,53$ & 0,398 \\
\hline \multicolumn{8}{|l|}{ Terceira etapa } \\
\hline Amamentação filhos anteriores $<6$ meses & 34 & 0,54 & 0,$16 ; 1,87$ & 0,333 & 1,24 & 0,$54 ; 2,82$ & 0,615 \\
\hline Cesárea & 60 & 1,53 & 0,$52 ; 4,51$ & 0,438 & 1,37 & 0,$59 ; 3,12$ & 0,460 \\
\hline \multicolumn{8}{|l|}{ Quarta etapa } \\
\hline Uso de chupeta aos 7 dias & 100 & 1,65 & 0,$79 ; 3,43$ & 0,180 & 1,90 & 1,$06 ; 3,44$ & $\mathbf{0 , 0 3 3}$ \\
\hline Trauma mamilar na maternidade & 92 & 2,13 & 1,$03 ; 4,42$ & 0,043 & - & - & - \\
\hline Trauma mamilar aos 7 dias & 99 & - & - & - & 2,07 & 1,$17 ; 3,68$ & 0,013 \\
\hline Ingurgitamento mamário aos 7 dias & 73 & 1,19 & 0,$57 ; 2,48$ & 0,648 & 1,31 & 0,$71 ; 2,43$ & 0,382 \\
\hline
\end{tabular}

Valores em negrito: associações estatsiticamente significantes a $\mathrm{p}<0,05$

* OR ajustada para as variáveis contidas no modelo

** Incluído na etapa seguinte

As Tabelas 4 e 5 apresentam as freqüências dos itens desfavoráveis aos 30 dias quanto à técnica na maternidade e quanto à técnica aos 30 dias, respectivamente. Nenhum dos itens pesquisados na maternidade se mostrou associado ao uso de mamadeira aos 30 dias. Por outro lado, os itens desfavoráveis aos 30 dias foram significativamente mais freqüentes entre as duplas cujas crianças faziam uso de mamadeira: cabeça e tronco da criança não alinhados, queixo da criança não toca a mama, boca pouco aberta e pega não assimétrica.

\section{DISCUSSÃO}

O presente estudo confirmou que o uso de mamadeira já no primeiro mês de vida da criança é bastante comum, principalmente para oferecer chás e água, apesar de a criança amamentada não precisar receber suplementação hídrica nos primeiros seis meses, mesmo em locais secos e quentes. ${ }^{1} \mathrm{O}$ uso de chás é prática arraigada na cultura brasileira, embora existam variações regionais. $\mathrm{Na}$ região Nordeste do Brasil foi constatado uso de chá e/ou água no primeiro mês em $88 \%$ das crianças, das quais $80 \%$ recebiam esses líquidos já na primeira semana. ${ }^{8} \mathrm{Na}$ mesma região, em outro estado, 23,2\% das crianças amamentadas no primeiro mês de vida recebiam chás, água $(7,3 \%)$ e suco $(2,7 \%){ }^{21}$ Embora no presente estudo a freqüência de uso de chá tenha sido elevada, contata-se diminuição dessa prática ao compará-la a estudo feito com a mesma população há 16 anos: de $69,4 \%{ }^{5}$ para $37 \%$. Isso provavelmente se deve aos esforços de diversos segmentos da sociedade e setor público visando à promoção do aleitamento materno, inclusive da amamentação exclusiva por seis meses.

Tabela 3. Número de itens desfavoráveis à técnica de amamentação e uso de mamadeira aos 30 dias de vida. Porto Alegre, RS, 2003.

\begin{tabular}{lccc}
\hline Escore & $\begin{array}{c}\text { Sim } \\
(\mathrm{N}=90) \\
\text { Média } \pm \mathrm{DP}\end{array}$ & $\begin{array}{c}\text { Não } \\
(\mathrm{N}=113)\end{array}$ & $\mathrm{p}^{*}$ \\
& & & \\
\hline Na maternia $\pm \mathrm{DP}$ & \\
$\quad \begin{array}{ccc}\text { Postura } \\
\quad \text { Pega da criança }\end{array}$ & $1,5 \pm 0,6$ & $1,4 \pm 0,7$ & 0,754 \\
Aos 30 dias** & & & \\
$\quad$ Postura & $2,1 \pm 1,1$ & $1,8 \pm 1,2$ & 0,043 \\
$\quad$ Pega da criança & $1,3 \pm 0,6$ & $1,0 \pm 0,6$ & 0,001 \\
\hline
\end{tabular}

* Teste t-student

** Excluídas 8 duplas que interromperam o aleitamento materno no primeiro mês 
Tabela 4. Freqüência de itens desfavoráveis à mamada na maternidade de acordo com o uso de mamadeira aos 30 dias. Porto Alegre, RS, 2003. ( $\mathrm{N}=203)$

\begin{tabular}{|c|c|c|c|c|c|}
\hline \multirow{3}{*}{ Técnica na maternidade } & \multicolumn{4}{|c|}{ Uso de mamadeira aos 30 dias } & \multirow{3}{*}{$\mathrm{p}^{*}$} \\
\hline & \multicolumn{2}{|c|}{ Sim } & \multicolumn{2}{|c|}{ Não } & \\
\hline & $N=90$ & $\%$ & $\mathrm{~N}=113$ & $\%$ & \\
\hline \multicolumn{6}{|l|}{ Posicionamento mãe/criança } \\
\hline Mãe com ombros tensos & 38 & 38,8 & 41 & 36,3 & 0,709 \\
\hline Criança distante da mãe & 14 & 14,3 & 15 & 13,3 & 0,831 \\
\hline Cabeça e corpo da criança não alinhados & 70 & 71,4 & 75 & 66,4 & 0,429 \\
\hline Queixo da criança não toca a mama & 22 & 22,4 & 26 & 23,0 & 0,923 \\
\hline Criança não apoiada adequadamente & 34 & 34,7 & 30 & 26,5 & 0,199 \\
\hline \multicolumn{6}{|l|}{ Pega da criança } \\
\hline Boca pouco aberta & 23 & 23,5 & 31 & 27,4 & 0,510 \\
\hline Lábio inferior não evertido & 22 & 22,4 & 19 & 16,8 & 0,302 \\
\hline Pega não assimétrica & 97 & 99,0 & 111 & 98,2 & 0,646 \\
\hline
\end{tabular}

* Teste qui-quadrado de Pearson

A pouca idade materna foi identificada como fator de risco para interrupção precoce do AME em dois estudos brasileiros, ${ }^{18,19}$ mas o presente estudo é o primeiro a identificar associação entre mãe adolescente e introdução de mamadeira na primeira semana de vida. Possivelmente as mães mais jovens sejam influenciadas pelas mulheres mais velhas, que tiveram filhos numa época em que era recomendada a oferta de líquidos à criança já nos primeiros dias de vida.

A coabitação com a avó materna da criança mostrou-se associada ao uso de mamadeira tanto aos sete quanto aos 30 dias de vida, mas não associada à coabitação com a avó paterna; provavelmente porque as mães parecem exercer maior influência sobre suas filhas do que sobre suas noras. Estudo anterior realizado no mesmo local da presente pesquisa mostrou que quando a avó materna aconselhava o uso de água e/ou chá no primeiro mês a chance de a criança não ser amamentada exclusivamente no primeiro mês aumentava 2,2 vezes. ${ }^{15}$ A maioria das avós das crianças do presente estudo teve filhos entre as décadas de 60 e 80, quando o aleitamento materno não era tão valorizado, em especial o exclusivo. As taxas de aleitamento materno nessa época eram baixas, o uso de água e chás era recomendado pelos pediatras e imperava a crença do "leite fraco" ou "pouco leite". Portanto, ao recomendarem o uso de água, chás ou mesmo outros leites, as avós transmitem às suas filhas a sua experiência, acreditando ser o mais adequado. Isso reforça a necessidade de se incluir as avós em programas de promoção do aleitamento materno para que práticas que se tornaram obsoletas nos últimos 20 anos não continuem sendo transmitidas às novas gerações de mães.

Tabela 5. Freqüência de itens desfavoráveis à mamada aos 30 dias de acordo com o uso de mamadeira aos 30 dias. Porto Alegre, RS, 2003. ( $\mathrm{N}=203)$

\begin{tabular}{|c|c|c|c|c|c|}
\hline \multirow{3}{*}{ Técnica aos 30 dias } & \multicolumn{4}{|c|}{ Uso de mamadeira aos 30 dias } & \multirow{3}{*}{$p^{*}$} \\
\hline & \multicolumn{2}{|c|}{ Sim } & \multicolumn{2}{|c|}{ Não } & \\
\hline & $\mathrm{N}=90$ & $\%$ & $N=113$ & $\%$ & \\
\hline \multicolumn{6}{|l|}{ Posicionamento mãe/criança } \\
\hline Mãe com ombros tensos & 38 & 42,2 & 44 & 38,9 & 0,636 \\
\hline Criança distante da mãe & 17 & 18,9 & 21 & 18,6 & 0,956 \\
\hline Cabeça e corpo da criança não alinhados & 65 & 72,2 & 61 & 54,0 & 0,008 \\
\hline Queixo da criança não toca a mama & 21 & 23,3 & 12 & 10,6 & 0,015 \\
\hline Criança não apoiado adequadamente & 48 & 53,3 & 62 & 54,9 & 0,828 \\
\hline \multicolumn{6}{|l|}{ Pega da criança } \\
\hline Boca pouco aberta & 21 & 23,3 & 8 & 7,1 & 0,001 \\
\hline Lábio inferior não evertido & 12 & 13,3 & 9 & 8,0 & 0,212 \\
\hline Pega não assimétrica & 85 & 94,4 & 97 & 85,8 & 0,046 \\
\hline
\end{tabular}

* Teste qui-quadrado de Pearson 
No presente estudo e em outro realizado no Nordeste brasileiro, ${ }^{8}$ o uso de chupeta aos sete dias mostrou-se associado ao uso de mamadeira ao final do primeiro mês. É possível que o uso de chupeta resulte em diminuição do número de mamadas e, conseqüentemente, em menor estímulo das mamas e redução da produção de leite,,$^{2,4,14}$ levando a mãe a oferecer mamadeira com leite não humano para saciar a fome da criança. Para alguns autores, o uso de chupeta seria um marcador de dificuldades no aleitamento materno e não o causador direto do desmame. ${ }^{7,20}$ Não se pode descartar, também, a possibilidade de que essa associação se deva ao fato de que as mães já conscientizadas quanto aos malefícios do hábito da chupeta também o sejam quanto ao uso da mamadeira, passando a evitá-la.

Trauma mamilar na maternidade e aos sete dias mostrou-se associado, respectivamente, ao uso de mamadeira aos sete e aos 30 dias de vida. A associação entre problemas precoces com os mamilos e introdução de leite não humano no primeiro mês também foi encontrada em estudo realizado no Nordeste. ${ }^{8}$ Muitas mães com trauma mamilar, para sentirem menos dor, oferecem mamadeira aos seus filhos para espaçarem as mamadas. Essa prática pode resultar em menor produção de leite, fazendo com que a oferta de mamadeira se perpetue.

Considerando que o oferecimento de qualquer líquido diferente do leite materno, em geral, é feito por mamadeira, pode-se afirmar que o presente estudo não constatou associação entre técnica de amamentação na maternidade e prevalências de AME no primeiro mês, descrita em outros estudos. Na Suécia ${ }^{12}$ foi relatado que as crianças com pega incorreta (sucção apenas do mamilo) no dia da alta da maternidade tinham uma chance dez vezes maior de receber mamadeira no primeiro mês quando comparadas com as crianças com pega correta ou que tiveram a sua pega corrigida na maternidade. Na Argentina ${ }^{3}$ foi constatado que as crianças com técnica de sucção considerada muito boa (criança abocanha bem a mama, com boca bem aberta, língua abaixo da aréola, lábio inferior evertido, suç̧ões com movimentos lentos e profundos) foram amamentados exclusivamente (leite materno e água) por quatro meses, em média. Já os considerados com técnica de sucção boa (criança abocanha a mama com dificuldade, mas suga corretamente após ter iniciado e adquirido uma boa sucção rítmica) mamaram exclusivamente por 3,5 meses; os com suç̧ão considerada ruim (crianças posicionadas muito "alto" na mama, boca pouco aberta, segura o mamilo apenas parcialmente e não consegue abocanhar a aréola), por apenas dois meses. ${ }^{3}$

$\mathrm{O}$ fato de o presente estudo ter mostrado associação entre uso de mamadeira e técnica de amamentação inadequada aos 30 dias, e não na maternidade, sugere que o uso de mamadeira influencia a técnica de amamentação, e não o contrário. Segundo Righard, ${ }^{13}$ as crianças com alimentação mista (peito e mamadeira) podem desenvolver técnica incorreta de sucção no seio. Para esse autor, algumas dessas crianças usam a língua como pistão ao sugar o seio, comportamento usual na sucção da mamadeira, mas não na suç̧ão do seio. $\mathrm{O}$ presente estudo corrobora com essa hipótese, já que as crianças que usavam mamadeira apresentaram com mais freqüência comportamentos desfavoráveis a uma transferência efetiva do leite da mama.

Além de apontar determinantes do uso precoce de mamadeira, o presente estudo é o primeiro a mostrar que, além dos efeitos negativos já conhecidos, a mamadeira pode influenciar negativamente a técnica de amamentação. O uso de chá ou água por mamadeira, prática tida como inofensiva por muitas mães e profissionais de saúde, pode contribuir para a introdução precoce de leites industrializados, pois a boa técnica de amamentação está relacionada com esvaziamento efetivo da mama. ${ }^{13}$ Não havendo essa condição, as mães tendem a produzir menos leite e as crianças a mamar com mais freqüência levando, muitas vezes, à complementação com outros leites. Portanto, se os resultados do presente estudo forem confirmados por estudos realizados em outras populações, o efeito negativo da sucção da mamadeira na técnica de amamentação pode ser mais um argumento contra a introdução de mamadeira em crianças que estão sendo amamentadas. 


\section{REFERÊNCIAS}

1. Ashraf RN, Jalil F, Aperia A, Lindblad BS. Additional water is not needed for healthy breast-fed babies in a hot climate. Acta Paediatr. 1993;82(12):1007-11. doi:10.1111/j.1651-2227.1993.tb12799.x

2. Barros, FC, Victora CG, Semer TC, Tonioli Filho S, Tomasi E, Weiderpass E. Use of pacifiers is associated with decreased breast-feeding duration. Pediatrics. 1995;95(4):479-99.

3. Cernadas JMC, Noceda G, Barrera L, Martinez AM, Garsd A. Maternal and perinatal factors influencing the duration of exclusive breastfeeding during the first 6 months of life. J Hum Lact. 2003;19(2):136-44. doi:10.1177/0890334403253292

4. Cunha AJLA, Leite AM, Machado MMT. Breastfeeding and pacifier use in Brazil. Indian J Pediatr. 2005;72(3):13-6. doi:10.1007/BF02859257

5. Issler RMS, Giugliani ERJ, Sefrin CF, Justo EB, Carvalho NM, Hartmann RM. Hábitos alimentares no primeiro ano de vida: estudo de uma coorte de crianças nascidas no Hospital de Clínicas de Porto Alegre. Rev Hosp Clin Porto Alegre. 1990;10:141-5.

6. Karjalainen S, Ronning O, Lapinleimu H, Simell O. Association between early weaning, non-nutritive sucking habits and occlusal anomalies in 3-year-old finnish children. Int J Paediatr Dent. 1999;9(3):169-73. doi:10.1046/j.1365-263x.1999.00133.x

7. Kramer MS, Barr RG, Degenais S, Yang H, Jones $P$, Ciofani L, et al. Pacifier use, early weaning, and cry/fuss behavior: a randomized controlled trial. JAMA. 2001;286(3):322-6. doi:10.1001/jama.286.3.322

8. Marques NM, Lira PIC, Lima MC, Silva NL, Batista Filho M, Huttly SRA, et al. Breastfeeding and early weaning practices in Northeast Brazil: a longitudinal study. Pediatrics. 2001;108(4):e66. doi:10.1542/ peds.108.4.e66

9. Motta VT, Wagner MB. Bioestatística. Caxias do Sul: Educs; 2003.

10. Neifert M, Lawrence R, Seacat J. Nipple confusion: toward a formal definition. J Pediatrics. 1995;126(6):125-9.

11. Palmer B. Influence of breastfeeding on the development of the oral cavity. J Hum Lact. 1998;14(2):93-8. doi:10.1177/089033449801400203
12. Righard L, Alade MO. Sucking technique and its effect on success of breastfeeding. Birth. 1992;19(4):185-9. doi:10.1111/j.1523-536X.1992.tb00399.x

13. Righard L. Early enhancement of successful breastfeeding. World Health Forum. 1996;17(1):92-7.

14. Soares MEM, Giugliani ERJ, Braun ML, Salgado ACN, Oliveira AP, Aguiar PR. Uso de chupeta e sua relação com o desmame precoce em população de crianças nascidas em Hospital Amigo da Criança. J Pediatr (Rio J). 2003;79(4):309-16. doi:10.1590/S002175572003000400008

15. Susin LRO, Giugliani ERJ. Influência das avós na prática do aleitamento materno. Rev Saude Publica. 2005;39(2):141-7. doi:10.1590/S003489102005000200001

16. Tully SB, Bar-Haim Y, Bradley RL. Abnormal tympanography after supine bottle feeding. I Pediatr. 1995;126(6):S105-11.

17. UNICEF; World Health Organization. Breastfeeding management and promotion in a Baby-Friendly Hospital: an 18 hour course for maternity staff. New York; 1993.

18. Venâncio SI, Escuder MML, Kitoko P, Rea MF, Monteiro CA. Freqüência e determinantes do aleitamento materno em municípios do Estado de São Paulo. Rev Saude Publica. 2002;36:313-8.

19. Venâncio SI, Monteiro CA. Individual and contextual determinants of exclusive breastfeeding in São Paulo, Brazil: a multilevel analysis. Public Health Nutr. 2006;9(1):40-6.

20. Victora CG, Behague DP, Barros FC, Olinto MTA, Weiderpass E. Pacifier use and short breastfeeding duration: cause, consequence, or coincidence? Pediatrics. 1997;99(3):445-53. doi:10.1542/ peds.99.3.445

21. Vieira GO, Almeida JAG, Silva LR, Cabral VA, Netto PVS. Fatores associados ao aleitamento materno e desmame em Feira de Santana, Bahia. Rev Bras Saude Matern Infant. 2004;4(2):143-50. doi:10.1590/S151938292004000200004

22. Viggiano D, Fasano D, Monaco G, Strohmenger L. Breast feeding, bottle feeding, and non-nutritive sucking; effects on occlusion in deciduous dentition. Arch Dis Child. 2004;89(12):1121-3. doi:10.1136/ adc. 2003.029728

Artigo baseado na dissertação de mestrado de MCT França, apresentada ao Programa de Pós-Graduação em Ciências Médicas: Pediatria, da Universidade Federal do Rio Grande do Sul, em 2005. 